\title{
Marine algal flora of Oho-ri, Gosung-gun, Gangwon-do, Korea
}

\author{
Eun Hee Bae ${ }^{1, *}$, Jung Hyun Oak² and Eun-Young Lee ${ }^{1}$ \\ ${ }^{1}$ National Institute of Biological Resources, Incheon 404-708, Korea \\ 2\#814, Department Oceanography, Pusan National University, 2, Busandaehak-ro 63beon-gil, Geumjeong-gu, Busan \\ 609-735, Korea \\ *Correspondent: ehbae@korea.kr
}

\begin{abstract}
It was found that a total of 94 species of marine algae, 13 green algae, 24 brown algae, and 57 red algae, are living at the seashore of Oho-ri in this study. 739 voucher specimens for 94 species were made based on plants from March to September 2010 at the 3 islets, including Heukdo, Goraebawi, and Juckdo. Voucher specimens were catalogued and deposited in the herbarium of the National Institute of Biological Resources (KB) of Korea.
\end{abstract}

Keywords: marine algal flora, Oho-ri, species list, voucher specimens

\section{INTRODUCTION}

We report the marine algal flora at the seashore of Ohori, Gosung-gun, Gangwon-do in this note. The seashore of Gosung-gun has predominantly sandy substrate with some rocky outcrops. The algal habitat of the eastern coast of Korea is generally restricted because of the steep slope and small tidal range.

The survey on flora was performed at 3 islets (the sea surface-protruding rocky outcrops), including Heukdo, Goraebawi, and Juckdo. The marine algal vegetation of Juckdo had reported (Koh, 1983; Koh and Sung, 1983). It had mentioned 42 species in the community research on marine algae from July 1982 to June 1983.

This study aimed to establish an inventory of algal species inhabiting Oho-ri coast nearby the eastern NLL (the Northern Limit Line), Gangwon-do, Korea. This area was a selected region for the project "The Survey on Species Diversity of Strategic Regions (2010)" in National Institute of Biological Resources (NIBR).

\section{Materials ANd Methods}

The field collections were conducted bimonthly from March to September in 2010. We selected three survey sites, Heukdo, Goraebawi, and Juckdo, which has abundant growth of marine algae (Fig. 1). The details of the SCUBA diving stations for collection are shown in
Table 1.

The collected samples were immediately preserved as dried specimen at the site or moved to the laboratory in cooled ice box. Standardized specimen preservation was used, according to the NIBR manual (NIBR, 2009). Specimens were identified on the basis of description by Kang (1968), Lee (2008) and Yoshida (1998) and were registered at the NIBR Biological Resources Management System by serial number. The specimens were stored at the herbarium of plant storage center of the NIBR International Standards Herbarium (KB).

\section{Results ANd Discussion}

\section{Habitat Description}

The deepest depths at which algae grow was $-24 \mathrm{~m}$ in Heukdo (St. 1-2) and $-20.6 \mathrm{~m}$ in Goraebawi (St. 3), $-13.3 \mathrm{~m}$ in Juckdo (St. 4-7), respectively. The seawater temperature was $8-10^{\circ} \mathrm{C}$ in May, $8-18^{\circ} \mathrm{C}$ in July, and $12-$ $22^{\circ} \mathrm{C}$ in September (Table 1). The year in which the surveys were conducted recorded lower temperatures than those in the previous year.

\section{Marine algal flora}

A total of 94 species belonging to the 21 orders, 35 families, and 67 genera were identified in this study. In all, 739 voucher specimens were obtained, and their inventory is as follows. 


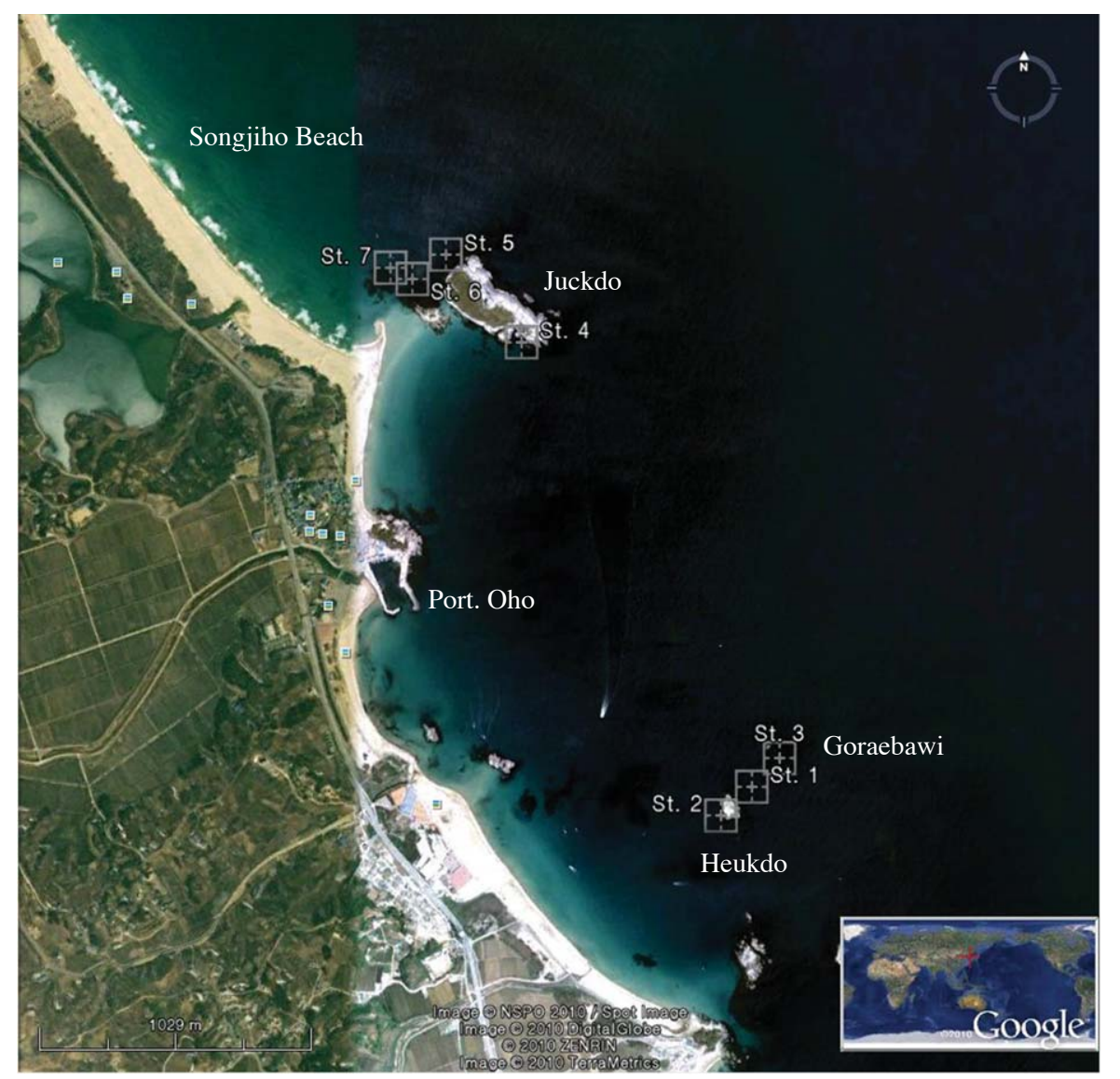

Fig. 1. Map denoting 3 islets and its diving stations where the survey was conducted.

Table 1. Details of marine algae survey station of Oho-ri coast, Gosung-gun.

\begin{tabular}{|c|c|c|c|c|c|}
\hline Locality & Station & GPS & Collection date & Max. of depth (m) & $\begin{array}{c}\text { Seawater } \\
\text { temperature }\left({ }^{\circ} \mathrm{C}\right)\end{array}$ \\
\hline \multirow{8}{*}{ Heukdo } & \multirow{4}{*}{ St. 1} & & 30-Mar-10 & 23 & - \\
\hline & & N $38^{\circ} 19^{\prime} 09.3^{\prime \prime}$ & 18-May-10 & 23.2 & 8 \\
\hline & & E $128^{\circ} 32^{\prime} 42.4^{\prime \prime}$ & 22-Jul-10 & 23.4 & 8 \\
\hline & & & 14-Sep-10 & 24 & 12 \\
\hline & \multirow{4}{*}{ St. 2} & & 30-Mar-10 & 15 & - \\
\hline & & N $38^{\circ} 19^{\prime} 05.8^{\prime \prime}$ & 18-May-10 & 14.8 & 10 \\
\hline & & E $128^{\circ} 32^{\prime} 37.5^{\prime \prime}$ & 22-Jul-10 & 14.9 & 11 \\
\hline & & & 14-Sep-10 & 15 & 19 \\
\hline \multirow{4}{*}{ Goraebawi } & \multirow{4}{*}{ St. 3} & & 30-Mar-10 & 18 & - \\
\hline & & N $38^{\circ} 19^{\prime} 12.8^{\prime \prime}$ & 18-May-10 & 20.6 & 9 \\
\hline & & E $128^{\circ} 32^{\prime} 46.8^{\prime \prime}$ & 21-Jul-10 & 18.7 & 10 \\
\hline & & & 14-Sep-10 & 20 & 12 \\
\hline \multirow{8}{*}{ Juckdo } & \multirow{3}{*}{ St. 4} & & 19-May-10 & 12.7 & 10 \\
\hline & & $\begin{array}{l}N 382003.5 \\
\text { F } 128^{\circ} 32^{\prime} 059^{\prime \prime}\end{array}$ & 21-Jul-10 & 13.3 & 16 \\
\hline & & E 1283205.9 & $15-$ Sep-10 & 13 & 17 \\
\hline & \multirow{2}{*}{ St. 5} & $\mathrm{~N} 38^{\circ} 20^{\prime} 14.2^{\prime \prime}$ & 31-Mar-10 & 11 & - \\
\hline & & E $128^{\circ} 31^{\prime} 53.9^{\prime \prime}$ & 19-May-10 & 10.1 & 10 \\
\hline & \multirow{2}{*}{ St. 6} & $\mathrm{~N} 38^{\circ} 20^{\prime} 11.2^{\prime \prime}$ & 21-Jul-10 & 10.8 & 18 \\
\hline & & E $128^{\circ} 31^{\prime} 48.6^{\prime \prime}$ & 15-Sep-10 & 8 & 22 \\
\hline & St. 7 & $\begin{array}{l}\text { N } 38^{\circ} 20^{\prime} 12.6^{\prime \prime} \\
\text { E } 128^{\circ} 31^{\prime} 45.1^{\prime \prime}\end{array}$ & 31-Mar-10 & 6 & - \\
\hline
\end{tabular}


Phylum Chlorophyta

Class Bryopsidophyceae

Order Bryopsidales

Family Bryopsidaceae

\section{Bryopsis hypnoides Lamouroux}

Examined specimens: St. 5; NIBRAL0000117289 (19 May 2010).

\section{Bryopsis plumosa (Hudson) C.Agardh}

Examined specimens: St. 3; NIBRAL0000121553 (14 Sep. 2010).

Family Codiaceae

\section{Codium arabicum Kützing}

Examined specimens: St. 1; NIBRAL0000117567 (22 Jul. 2010), NIBRAL0000121642 (14 Sep. 2010), NIBRAL0 000121643 (14 Sep. 2010). St. 2; NIBRAL000011 7124 (30 Mar. 2010), NIBRAL0000117626 (22 Jul. 2010), NI BRAL0000121644 (14 Sep. 2010), NIBRAL0000121645 (14 Sep. 2010). St. 3; NIBRAL000011 7281 (18 May 2010), NIBRAL0000121646 (14 Sep. 2010).

\section{Codium fragile (Suringar) Hariot}

Examined specimens: St. 1; NIBRAL0000117446 (18 May 2010). St. 2; NIBRAL0000117624 (22 Jul. 2010), NIBRAL0000121625 (14 Sep. 2010). St. 4; NIBRAL00 00121628 (15 Sep. 2010), NIBRAL0000121629 (15 Sep. 2010). St. 5; NIBRAL0000117444 (19 May 2010), NIB RAL0000117445 (19 May 2010). St. 6; NIBRAL000011 7625 (21 Jul. 2010), NIBRAL0000121626 (15 Sep. 2010), NIBRAL0000121627 (15 Sep. 2010).

\section{Class Ulvophyceae}

Order Cladophorales

Family Cladophoraceae

\section{Chaetomorpha moniligera Kjellman}

Examined specimens: St. 4; NIBRAL0000117285 (19 May 2010).

\section{Cladophora japonica Yamada}

Examined specimens: St. 1; NIBRAL0000117652 (22 Jul. 2010). St. 2; NIBRAL0000117651 (22 Jul. 2010). St. 3; NIBRAL0000117649 (21 Jul. 2010), NIBRAL0000117 650 (21 Jul. 2010).

\section{Cladophora sakaii Abbott}

Examined specimens: St. 2; NIBRAL0000117642 (22 Jul. 2010), NIBRAL0000117643 (22 Jul. 2010). St. 3; NIBR AL0000117641 (21 Jul. 2010). St. 5; NIBRAL0000117 782 (19 May 2010). St. 6; NIBRAL0000117639 (21 Jul. 2010), NIBRAL0000117640 (21 Jul. 2010).

\section{Cladophora stimpsonii Harvey}

Examined specimens: St. 5; NIBRAL0000117316 (19 May 2010), NIBRAL0000117317 (19 May 2010), NIBR AL0000117318 (19 May 2010).

\section{Order Ulotrichales}

Family Monostromataceae

\section{Monostroma grevillei (Thuret) Wittrock}

Examined specimens: St. 2; NIBRAL0000117119 (30 Mar. 2010), NIBRAL0000117120 (30 Mar. 2010). St. 3; NIBRAL0000117118 (30 Mar. 2010), NIBRAL000011 7192 (30 Mar. 2010). St. 5; NIBRAL0000117117 (31 Mar. 2010).

Order Ulvales

Family Ulvaceae

\section{Ulva compressa Linnaeus}

Examined specimens: St. 2; NIBRAL0000117113 (30 Mar. 2010).

\section{Ulva linza Linnaeus}

Examined specimens: St. 2; NIBRAL0000117102 (30 Mar. 2010).

\section{Ulva pertusa Kjellman}

Examined specimens: St. 1; NIBRAL0000117736 (22 Jul. 2010). St. 2; NIBRAL0000117109 (30 Mar. 2010), NIB RAL0000117110 (30 Mar. 2010), NIBRAL0000117111 (30 Mar. 2010), NIBRAL0000117377 (18 May 2010); NIBRAL0000117381 (18 May 2010), NIBRAL0000117 384 (18 May 2010), NIBRAL0000117737 (22 Jul. 2010), NIBRAL0000117741 (22 Jul. 2010), NIBRAL0000117 742 (22 Jul. 2010), NIBRAL0000117763 (22, Jul. 2010), NIBRAL0000121531 (14 Sep. 2010), NIBRAL0000121 532 (14 Sep. 2010), NIBRAL0000121533 (14 Sep. 2010). St. 3; NIBRAL0000117375 (18 May 2010), NIBRAL00 00117738 (21 Jul. 2010), NIBRAL0000117739 (21 Jul. 2010), NIBRAL0000117740 (21 Jul. 2010). St. 4; NIBR AL0000117369 (19 May 2010), NIBRAL0000117373 
(19 May 2010), NIBRAL0000117743 (21 Jul. 2010), NI BRAL0000121537 (15 Sep. 2010). St. 5; NIBRAL0000 117112 (31 Mar. 2010), NIBRAL0000117246 (31 Mar. 2010), NIBRAL0000117367 (19 May 2010), NIBRAL0 000117371 (19 May 2010). St. 6; NIBRAL0000117735 (21 Jul. 2010), NIBRAL0000121534 (15 Sep. 2010), NI BRAL0000121535 (15 Sep. 2010), NIBRAL000012153 6(15 Sep. 2010).

\section{Ulvaria obscura var. blyttii (Areschoug) Bliding}

Examined specimens: St. 1; NIBRAL0000117359 (18 May 2010). St. 2; NIBRAL0000117114 (30 Mar. 2010), NIBRAL0000117733 (22 Jul. 2010), NIBRAL0000117 734 (22 Jul. 2010). St. 3; NIBRAL0000117115 (30 Mar. 2010). St. 4; NIBRAL0000117362 (19 May 2010), NIB RAL0000117731 (21 Jul. 2010). St. 5; NIBRAL0000117 274 (19 May 2010), NIBRAL0000117349 (19 May 2010), NIBRAL0000117352 (19 May 2010), NIBRAL0000117 354 (19 May 2010), NIBRAL0000117357 (19 May 2010). St. 6; NIBRAL0000117729 (21 Jul. 2010), NIBRAL000 0117730 (21 Jul. 2010), NIBRAL0000117732 (21 Jul. 2010). St. 7; NIBRAL0000117116 (31 Mar. 2010).

Phylum Phaeophyta

Class Phaeophyceae

Order Desmarestiales

Family Desmarestiaceae

\section{Desmarestia viridis (O.F.Müller) J.V.Lamouroux}

Examined specimens: St. 1; NIBRAL0000117228 (30 Mar. 2010), NIBRAL0000117432 (18 May 2010), NIB RAL0000117433 (18 May 2010), NIBRAL0000117435 (18 May 2010), NIBRAL0000117629 (22 Jul. 2010), NI BRAL0000117630 (22 Jul. 2010), NIBRAL0000117631 (22 Jul. 2010), NIBRAL0000117796 (18 May 2010), NI BRAL0000121560 (14 Sep. 2010). St. 2; NIBRAL0000 117226 (30 Mar. 2010), NIBRAL0000117227 (30 Mar. 2010), NIBRAL0000117420 (18 May 2010), NIBRAL0 000117421 (18 May 2010), NIBRAL0000117422 (18 May 2010), NIBRAL0000117627 (22 Jul. 2010), NIBR AL0000117628 (22 Jul. 2010), NIBRAL0000117795 (18 May 2010). St. 3; NIBRAL0000117180 (30 Mar. 2010), NIBRAL0000117408 (18 May 2010), NIBRAL0000117 409 (18 May 2010), NIBRAL0000117410 (18 May 2010), NIBRAL0000117632 (21 Jul. 2010), NIBRAL0000117 633 (21 Jul. 2010), NIBRAL0000117634 (21 Jul. 2010), NIBRAL0000121562 (14 Sep. 2010), NIBRAL0000121 563 (14 Sep. 2010). St. 4; NIBRAL0000117405 (19 May 2010), NIBRAL0000117406 (19 May 2010), NIBRAL0 000117637 (21 Jul. 2010), NIBRAL0000117638 (21 Jul. 2010), NIBRAL0000121564 (15 Sep. 2010). St. 5; NIB RAL0000117225 (31 Mar. 2010), NIBRAL0000117400
(19 May 2010), NIBRAL0000117402 (19 May 2010), NIBRAL0000117403 (19 May 2010). St. 6; NIBRAL00 00117635 (21 Jul. 2010), NIBRAL0000117636 (21 Jul. 2010).
Order Dictyotales
Family Dictyotaceae

\section{Dictyopteris divaricata (Okamura) Okamura}

Examined specimens: St. 1; NIBRAL0000117385 (18 May 2010). St. 2; NIBRAL0000117380 (18 May 2010), NIBRAL0000117382 (18 May 2010), NIBRAL0000117 383 (18 May 2010), NIBRAL0000117724 (22 Jul. 2010), NIBRAL0000117725 (22 Jul. 2010), NIBRAL00001215 50 (14 Sep. 2010), NIBRAL0000117183 (30 Mar. 2010). St. 3; NIBRAL0000117378 (18 May 2010), NIBRAL00 00117379 (18 May 2010), NIBRAL0000117722 (21 Jul. 2010), NIBRAL0000117723 (21 Jul. 2010), NIBRAL00 00121551 (14 Sep. 2010). St. 4; NIBRAL0000117372 (19 May 2010), NIBRAL0000117374 (19 May 2010), NIBR AL0000117376 (19 May 2010), NIBRAL0000117727 (21 Jul. 2010), NIBRAL0000117728 (21 Jul. 2010), NI BRAL0000121552 (15 Sep. 2010). St. 5; NIBRAL0000 117275 (19 May 2010), NIBRAL0000117368 (19 May 2010), NIBRAL0000117370 (19 May 2010), NIBRAL0 000117185 (31 Mar. 2010), NIBRAL0000117186 (31 Mar. 2010). St. 6; NIBRAL0000117726 (21 Jul. 2010). St. 7; NIBRAL0000117184 (31 Mar. 2010).

\section{Dictyota dichotoma (Hudson) J.V.Lamouroux}

Examined specimens: St. 2; NIBRAL0000117562 (22 Jul. 2010), NIBRAL0000121540 (14 Sep. 2010). St. 3; NIB RAL0000117282 (18 May 2010), NIBRAL0000117561 (21 Jul. 2010). St. 6; NIBRAL0000117563 (21 Jul. 2010).

\section{Spatoglossum crassum J.Tanaka}

Examined specimens: St. 4; NIBRAL0000117744 (21 Jul. 2010), NIBRAL0000117745 (21 Jul. 2010).

\section{Spatoglossum pacificum Yendo}

Examined specimens: St. 1; NIBRAL0000117564 (22 Jul. 2010).

\section{Zonaria diesingiana J.Agardh}

Examined specimens: St. 1; NIBRAL0000121565 (14 Sep. 2010).

Order Ectocarpales

Family Chordariaceae 


\section{Leathesia difformis Areschoug}

Examined specimens: St. 2; NIBRAL0000117243 (18 May 2010), NIBRAL0000117552 (22 Jul. 2010), NIBR AL0000117553 (22 Jul. 2010) NIBRAL0000117554 (22 Jul. 2010). St. 4; NIBRAL0000117555 (21 Jul. 2010).

\section{Papenfussiella kuromo (Yendo) Inagaki}

Examined specimens: St. 4; NIBRAL0000117284 (19 May 2010).

Order Fucales

Family Sargassaceae

\section{Myagropsis myagroides (Mertens ex Turner) Fensholt}

Examined specimens: St. 2; NIBRAL0000117280 (18 May 2010). St. 4; NIBRAL0000117283 (19 May 2010). St. 5; NIBRAL0000117254 (31 Mar. 2010).

\section{Sargassum confusum C.Agardh}

Examined specimens: St. 1; NIBRAL0000117590 (22 Jul. 2010), NIBRAL0000117591 (22 Jul. 2010), NIBRA L0000117592 (22 Jul. 2010), NIBRAL0000117596 (22 Jul. 2010), NIBRAL0000121583 (14 Sep. 2010). St. 2; NIBRAL0000117595 (22 Jul. 2010). St. 3; NIBRAL000 0117593 (21 Jul. 2010), NIBRAL0000117594 (21 Jul. 2010), NIBRAL0000121674 (14 Sep. 2010), NIBRAL0 000121675 (14 Sep. 2010). St. 4; NIBRAL0000117597 (21 Jul. 2010), NIBRAL0000117598 (21 Jul. 2010).

\section{Sargassum hemiphyllum (Turner) C.Agardh}

Examined specimens: St. 1; NIBRAL0000121584 (14 Sep. 2010), NIBRAL0000121585 (14 Sep. 2010), NIBR AL0000121586(14 Sep. 2010). St. 2; NIBRAL0000121 587 (14 Sep. 2010). St. 3; NIBRAL0000121588 (14 Sep. 2010). St. 4; NIBRAL0000117588 (21 Jul. 2010), NIBR AL0000117589 (21 Jul. 2010), NIBRAL0000121591 (15 Sep. 2010). St. 6; NIBRAL0000121589 (15 Sep. 2010), NIBRAL0000121590 (15 Sep. 2010).

\section{Sargassum horneri (Turner) C.Agardh}

Examined specimens: St. 2; NIBRAL0000117568 (22 Jul. 2010), NIBRAL0000117764 (22 Jul. 2010), NIBRA L0000121597 (14 Sep. 2010). St. 3; NIBRAL00001215 98 (14 Sep. 2010). St. 4; NIBRAL0000121599 (15 Sep. 2010). St. 6; NIBRAL0000117586(21 Jul. 2010), NIBR AL0000117587 (21 Jul. 2010).

\section{Sargassum micracanthum (Kützing) Endlicher}

Examined specimens: St. 6; NIBRAL0000121592 (15 Sep. 2010), NIBRAL0000121593 (15 Sep. 2010).

\section{Sargassum miyabei Yendo}

Examined specimens: St. 1; NIBRAL0000121582 (14 Sep. 2010). St. 6; NIBRAL0000117528 (21 Jul. 2010).

\section{Sargassum yezoense (Yamada) Yoshida and T. Konno}

Examined specimens: St. 6; NIBRAL0000117529 (21 Jul. 2010).

Order Laminariales
Family Alariacea

\section{Undaria pinnatifida (Harvey) Suringar}

Examined specimens: St. 1; NIBRAL0000117693 (22 Jul. 2010), NIBRAL0000117765 (22 Jul. 2010), NIBRA L0000121538 (14 Sep. 2010). St. 2; NIBRAL00001171 99 (30 Mar. 2010), NIBRAL0000117245 (30 Mar. 2010), NIBRAL0000117691 (22 Jul. 2010), NIBRAL0000117 692 (22 Jul. 2010), NIBRAL0000117766 (22 Jul. 2010), NIBRAL0000117783 (18 May 2010), NIBRAL0000117 807 ( 22 Jul. 2010), NIBRAL0000117814 (18 May 2010), NIBRAL0000117815 (18 May 2010), NIBRAL0000121 539 (14 Sep. 2010). St. 3; NIBRAL0000117689 (21 Jul. 2010), NIBRAL0000117690 (21 Jul. 2010), NIBRAL00 00117808 (21 Jul. 2010). St. 4; NIBRAL0000117694 (21 Jul. 2010). St. 5; NIBRAL0000117805 (19 May 2010), NIBRAL0000117810 (31 Mar. 2010).

\section{Family Costariaceae}

\section{Agarum clathratum Dumortier}

Examined specimens: St. 1; NIBRAL0000117223 (30 Mar. 2010), NIBRAL0000117224 (30 Mar. 2010), NIB RAL0000117425 (18 May 2010), NIBRAL0000117697 (22 Jul. 2010), NIBRAL0000117698 (22 Jul. 2010), NI BRAL0000117776 (22 Jul. 2010), NIBRAL0000117777 (22 Jul. 2010), NIBRAL0000117794 (18 May 2010), NI BRAL0000117797 (30 Mar. 2010), NIBRAL00001216 00 (14 Sep. 2010), NIBRAL0000121601 (14 Sep. 2010). St. 2; NIBRAL0000117222 (30 Mar. 2010), NIBRAL00 00117241 (30 Mar. 2010), NIBRAL0000117778 (22 Jul. 2010), NIBRAL0000117793 (18 May 2010), NIBRAL0 000117799 (30 Mar. 2010), NIBRAL0000117806 (22 Jul. 2010), NIBRAL0000121602 (14 Sep. 2010), NIBRAL0 000121676 (14 Sep. 2010). St. 3; NIBRAL0000117219 
(30 Mar. 2010), NIBRAL0000117220 (30 Mar. 2010), NIBRAL0000117242 (30 Mar. 2010), NIBRAL0000117 423 (18 May 2010), NIBRAL0000117424 (18 May 2010), NIBRAL0000117695 (21 Jul. 2010), NIBRAL0000117 696 (21 Jul. 2010), NIBRAL0000117775 (21 Jul. 2010), NIBRAL0000121603 (14 Sep. 2010), NIBRAL0000121 604 (14 Sep. 2010). St. 4; NIBRAL0000117699 (21 Jul. 2010), NIBRAL0000121605 (15 Sep. 2010). St. 5; NIB RAL0000117218 (31 Mar. 2010).

\section{Costaria costata (C.Agardh) De A.Saunders}

Examined specimens: St. 1; NIBRAL0000117350 (18 May 2010), NIBRAL0000117682 (22 Jul. 2010), NIBR AL0000117683 (22 Jul. 2010), NIBRAL0000117781 (22 Jul. 2010), NIBRAL0000117788 (18 May 2010), NI BRAL0000117789 (18 May 2010), NIBRAL000011780 0 (30 Mar. 2010), NIBRAL0000117813 (30 Mar. 2010). St. 2; NIBRAL0000117104 (30 Mar. 2010), NIBRAL00 00117202 (30 Mar. 2010), NIBRAL0000117221 (30 Mar. 2010), NIBRAL0000117347 (18 May 2010), NIBRAL0 000117348 (18 May 2010), NIBRAL0000117787 (18 May 2010), NIBRAL0000117809 (30 Mar. 2010), NIBR AL0000117816(18 May 2010). St. 3; NIBRAL00001171 06 (30 Mar. 2010), NIBRAL0000117107 (30 Mar. 2010), NIBRAL0000117108 (30 Mar. 2010), NIBRAL000011 7346 (18 May 2010), NIBRAL0000117679 (21 Jul. 2010), NIBRAL0000117680 (21 Jul. 2010), NIBRAL0000117 681 (21 Jul. 2010), NIBRAL0000117779 (21 Jul. 2010), NIBRAL0000117780 (21 Jul. 2010), NIBRAL00001177 86 (18 May 2010), NIBRAL0000117801 (30 Mar. 2010), NIBRAL0000117811 (30 Mar. 2010). St. 4; NIBRAL00 00117344 (19 May 2010), NIBRAL0000117686 (21 Jul. 2010), NIBRAL0000117784 (19 May 2010), NIBRAL0 000117785 (19 May 2010), NIBRAL0000117817 (19 May 2010). St. 5; NIBRAL0000117201 (31 Mar. 2010), NIBRAL0000117341 (19 May 2010), NIBRAL0000117 343 (19 May 2010), NIBRAL0000117812 (31 Mar. 2010). St. 6; NIBRAL0000117684 (21 Jul. 2010), NIBRAL000 0117685 (21 Jul. 2010). St. 7; NIBRAL0000117105 (31 Mar. 2010).

Family Laminariaceae

\section{Saccharina japonica (Areschoug) C.E.Lane, C.Mayes, Druehl and G.W.Saunders}

Examined specimens: St. 1; NIBRAL0000117342 (18 May 2010), NIBRAL0000117675 (22 Jul. 2010), NIBRA L0000117676 (22 Jul. 2010), NIBRAL0000117769 (22 Jul. 2010), NIBRAL0000117770 (22 Jul. 2010), NIBRA L0000117792 (18 May 2010), NIBRAL0000121620 (14 Sep. 2010). St. 2; NIBRAL0000117332 (18 May 2010),
NIBRAL0000117333 (18 May 2010), NIBRAL0000117 335 (18 May 2010), NIBRAL0000117339 (18 May 2010), NIBRAL0000117345 (18 May 2010), NIBRAL0000117 672 (22 Jul. 2010), NIBRAL0000117673 (22 Jul. 2010), NIBRAL0000117674 (22 Jul. 2010), NIBRAL0000117 771 (22 Jul. 2010), NIBRAL0000117772 (22 Jul. 2010), NIBRAL0000117773 (22 Jul. 2010), NIBRAL0000117 774 (22 Jul. 2010), NIBRAL0000117791 (18 May 2010), NIBRAL0000121621 (14 Sep. 2010), NIBRAL0000121 622 (14 Sep. 2010). St. 3; NIBRAL0000117670 (21 Jul. 2010), NIBRAL0000117671 (21 Jul. 2010), NIBRAL00 00117767 (21 Jul. 2010), NIBRAL0000117768 (21 Jul. 2010), NIBRAL0000121623 (14 Sep. 2010), NIBRAL0 000121624 (14 Sep. 2010). St. 4; NIBRAL0000117324 (19 May 2010), NIBRAL0000117325 (19 May 2010), NI BRAL0000117327 (19 May 2010), NIBRAL000011779 0 (19 May 2010). St. 5; NIBRAL0000117320 (19 May 2010), NIBRAL0000117321 (19 May 2010). St. 6; NIB RAL0000117677 (21 Jul. 2010), NIBRAL0000117678 (21 Jul. 2010).

$$
\begin{aligned}
& \text { Order Scytosiphonales } \\
& \text { Family Scytosiphonaceae }
\end{aligned}
$$

\section{Colpomenia bullosa (De A.Saunders) Yamada}

Examined specimens: St. 2; NIBRAL0000117244 (30 Mar. 2010).

\section{Colpomenia sinuosa (Mertens ex Roth) Derbès and Solier in Castagne}

Examined specimens: St. 1; NIBRAL0000117366 (18 May 2010). St. 2; NIBRAL0000117197 (30 Mar. 2010), NIBRAL0000117198 (30 Mar. 2010), NIBRAL0000117 361 (18 May 2010), NIBRAL0000117363 (18 May 2010), NIBRAL0000117364 (18 May 2010), NIBRAL0000117 365 (18 May 2010), NIBRAL0000117560 (22 Jul. 2010). St. 3; NIBRAL0000117358 (18 May 2010), NIBRAL00 00117360 (18 May 2010), NIBRAL0000117556 (21 Jul. 2010), NIBRAL0000117558 (21 Jul. 2010). St. 4; NIBR AL0000117355 (19 May 2010), NIBRAL0000117356 (19 May 2010), NIBRAL0000117558 (21 Jul. 2010), NI BRAL0000117559 (21 Jul. 2010). St. 5; NIBRAL00001 17195 (31 Mar. 2010), NIBRAL0000117196 (31 Mar. 2010), NIBRAL0000117351 (19 May 2010), NIBRAL0 000117353 (19 May 2010).

\section{Hydroclathrus clathratus (C.Agardh) M.A.Howe in N.L. Britton and C.F. Millspaugh}

Examined specimens: St. 2; NIBRAL0000117279 (18 May 2010). 


\section{Scytosiphon lomentaria (Lyngbye) Link}

Examined specimens: St. 2; NIBRAL0000117278 (18 May 2010).

Order Sporochnales

Family Sporochnaceae

\section{Sporochnus radiciformis (R.Brown ex Turner) C.Agardh}

Examined specimens: St. 1; NIBRAL0000117125 (30 Mar. 2010).

Phylum Rhodophyta

Class Bangiophyceae

Order Bangiales

Family Bangiaceae

38. Pyropia tenera (Kjellman) N.Kikuchi \& M.Miyata, M.S. Hwang \& H.G.Choi (as Porphyra tenera Kjellman)

Examined specimens: St. 4; NIBRAL0000117300 (19 May 2010), NIBRAL0000117301 (19 May 2010). St. 5; NIBRAL0000117190 (31 Mar. 2010), NIBRAL0000117 290 (19 May 2010), NIBRAL0000117299 (19 May 2010). St. 7; NIBRAL0000117189 (31 Mar. 2010).

Class Florideophyceae

Order Bonnemaisoniales

Family Bonnemaisoniaceae

\section{Bonnemaisonia hamifera Hariot}

Examined specimens: St. 1; NIBRAL0000117251 (30 Mar. 2010), NIBRAL0000117454 (18 May 2010), NIB RAL0000117455 (18 May 2010), NIBRAL0000117456 (18 May 2010), NIBRAL0000117601 (22 Jul. 2010), NI BRAL0000117602 (22 Jul. 2010), NIBRAL0000121639 (14 Sep. 2010). St. 2; NIBRAL0000117452 (18 May 2010), NIBRAL0000117453 (18 May 2010), NIBRAL0 000117600 (22 Jul. 2010). St. 3; NIBRAL0000117248 (30 Mar. 2010), NIBRAL0000117288 (18 May 2010), NIBRAL0000117451 (19 May 2010), NIBRAL0000117 599 (21 Jul. 2010), NIBRAL0000121640 (14 Sep. 2010), NIBRAL0000121641 (14 Sep. 2010). St. 4; NIBRAL00 00117449 (19 May 2010), NIBRAL0000117450 (19 May 2010). St. 5; NIBRAL0000117249 (31 Mar. 2010), NIB RAL0000117250 (31 Mar. 2010), NIBRAL0000117441 (19 May 2010), NIBRAL0000117447 (19 May 2010), NIBRAL0000117448 (19 May 2010). St. 6; NIBRAL00 00117603 (21 Jul. 2010).

\section{Delisea pulchra (Greville) Montagne}

Examined specimens: St. 2; NIBRAL0000117313 (18 May 2010). St. 3; NIBRAL0000117194 (30 Mar. 2010). St. 5; NIBRAL0000117193 (31 Mar. 2010), NIBRAL00 00117312 (19 May 2010).

Order Ceramiales

Family Callithamniaceae

\section{Aglaothamnion oosumiense Itono}

Examined specimens: St. 3; NIBRAL0000121549 (14 Sep. 2010). St. 4; NIBRAL0000117340 (19 May 2010), NIBRAL0000117531 (21 Jul. 2010). St. 5; NIBRAL000 0117338 (19 May 2010).

Family Ceramiaceae

\section{Ceramium boydenii E.S.Gepp}

Examined specimens: St. 6; NIBRAL0000117687 (21 Jul. 2010), NIBRAL0000117688 (21 Jul. 2010).

\section{Herpochondria elegans (Okamura) Itono}

Examined specimens: St. 4; NIBRAL0000117322 (18 May 2010). St. 5; NIBRAL0000117101 (31 Mar. 2010).

\section{Pterothamnion yezoense (Inagaki) Athanasiadis and Kraft}

Examined specimens: St. 2; NIBRAL0000117323 (18 May 2010). St. 5; NIBRAL0000117182 (31 Mar. 2010), NIBRAL0000117273 (19 May 2010), NIBRAL0000117 287 (19 May 2010).

Family Dasyaceae

\section{Dasya collabens J.D.Hooker and Harvey}

Examined specimens: St. 7; NIBRAL0000117187 (31 Mar. 2010).

\section{Dasya villosa Harvey}

Examined specimens: St. 4; NIBRAL0000117314 (19 May 2010), NIBRAL0000117315 (19 May 2010).

\section{Heterosiphonia japonica Yendo}

Examined specimens: St. 1; NIBRAL0000117618 (22 Jul. 2010), NIBRAL0000117619 (22 Jul. 2010). St. 2; NI BRAL0000117616 (22 Jul. 2010), NIBRAL0000117617 
(22 Jul. 2010), NIBRAL0000121638 (14 Sep. 2010). St. 3; NIBRAL0000117188 (30 Mar. 2010), NIBRAL0000 117336 (18 May 2010), NIBRAL0000117337 (18 May 2010), NIBRAL0000117614 (21 Jul. 2010), NIBRAL00 00117615 (21 Jul. 2010). St. 4; NIBRAL0000117622 (21 Jul. 2010), NIBRAL0000117623 (21 Jul. 2010). St. 5; NI BRAL0000117276 (19 May 2010). St. 6; NIBRAL0000 117620 (21 Jul. 2010), NIBRAL0000117621 (21 Jul. 2010).

\section{Heterosiphonia pulchra (Okamura) Falkenberg}

Examined specimens: St. 1; NIBRAL0000117252 (30 Mar. 2010), NIBRAL0000117613 (22 Jul. 2010). St. 2; NIBRAL0000117181 (30 Mar. 2010), NIBRAL0000117 27 (18 May 2010), NIBRAL0000121595 (14 Sep. 2010). St. 3; NIBRAL0000117271 (18 May 2010), NIBRAL00 00117664 (21 Jul. 2010), NIBRAL0000121548 (14 Sep. 2010). St. 4; NIBRAL0000121596(15 Sep. 2010). St. 5; NIBRAL0000117270 (19 May 2010). St. 6; NIBRAL00 00117612 (21 Jul. 2010).

Family Delesseriaceae

\section{Acrosorium yendoi Yamada}

Examined specimens: St. 1; NIBRAL0000121570 (14 Sep. 2010), NIBRAL0000121571 (14 Sep. 2010). St. 3; NIBRAL0000117667 (21 Jul. 2010), NIBRAL0000117 668 (21 Jul. 2010), NIBRAL0000121572 (14 Sep. 2010). St. 6; NIBRAL0000121573 (15 Sep. 2010).

\section{Delesseria serrulata Harvey}

Examined specimens: St. 1; NIBRAL0000117217 (30 Mar. 2010). St. 2; NIBRAL0000117214 (30 Mar. 2010), NIBRAL0000117215 (30 Mar. 2010), NIBRAL000011 7216 (30 Mar. 2010), NIBRAL0000117297 (18 May 2010), NIBRAL0000117298 (18 May 2010), NIBRAL0 000117798 (30 Mar. 2010). St. 3; NIBRAL0000117213 (30 Mar. 2010). St. 4; NIBRAL0000117294 (19 May 2010), NIBRAL0000117296 (19 May 2010), NIBRAL0 000117803 (19 May 2010). St. 5; NIBRAL0000117212 (31 Mar. 2010), NIBRAL0000117293 (19 May 2010), NIBRAL0000117295 (19 May 2010), NIBRAL0000117 802 (19 May 2010). St. 7; NIBRAL0000117211 (31 Mar. 2010).

\section{Erythroglossum minimum Okamura}

Examined specimens: St. 1; NIBRAL0000117329 (18 May 2010), NIBRAL0000117661 (22 Jul. 2010). St. 2; NIBRAL0000117328 (18 May 2010), NIBRAL0000121 574 (14 Sep. 2010). St. 3; NIBRAL0000117191 (30 Mar.
2010), NIBRAL0000117191 (30 Mar. 2010).

\section{Neoholmesia japonica(Okamura) Mikami}

Examined specimens: St. 1; NIBRAL0000117238 (30 Mar. 2010), NIBRAL0000117239 (30 Mar. 2010), NIB RAL0000117240 (30 Mar. 2010), NIBRAL0000117401 (18 May 2010), NIBRAL0000117404 (18 May 2010), NIBRAL0000117705 (22 Jul. 2010), NIBRAL0000117 706 (22 Jul. 2010), NIBRAL0000121633 (14 Sep. 2010), NIBRAL0000121634 (14 Sep. 2010). St. 2; NIBRAL00 00117236 (30 Mar. 2010), NIBRAL0000117237 (30 Mar. 2010), NIBRAL0000117393 (18 May 2010), NIBRAL0 000117396 (18 May 2010), NIBRAL0000117399 (18 May 2010), NIBRAL0000117703 (22 Jul. 2010), NIBR AL0000117704 (22 Jul. 2010), NIBRAL0000121635 (14 Sep. 2010). St. 3; NIBRAL0000117233 (30 Mar. 2010), NIBRAL0000117234 (30 Mar. 2010), NIBRAL000011 7235 (30 Mar. 2010), NIBRAL0000117386 (18 May 2010), NIBRAL0000117389 (18 May 2010), NIBRAL0 000117390 (18 May 2010), NIBRAL0000117700 (21 Jul. 2010), NIBRAL0000117701 (21 Jul. 2010), NIBRAL00 00117702 (21 Jul. 2010), NIBRAL0000121636 (14 Sep. 2010), NIBRAL0000121637 (14 Sep. 2010).

\section{Sorella repens (Okamura) Hollenberg}

Examined specimens: St. 3; NIBRAL0000117663 (21 Jul. 2010).

Family Rhodomelaceae

\section{Chondria crassicaulis Harvey}

Examined specimens: St. 1; NIBRAL0000117103 (30 Mar. 2010), NIBRAL0000117538 (22 Jul. 2010), NIBR AL0000117539(22 Jul. 2010). St. 3; NIBRAL00001215 80 (14 Sep. 2010). St. 4; NIBRAL0000117277 (19 May 2010), NIBRAL0000117611 (21 Jul. 2010), NIBRAL00 00121581 (15 Sep. 2010).

\section{Laurencia intricata J.V.Lamouroux}

Examined specimens: St. 4; NIBRAL0000117540 (21 Jul. 2010), NIBRAL0000117604 (21 Jul. 2010).

\section{Laurencia nipponica Yamada}

Examined specimens: St. 6; NIBRAL0000117579 (21 Jul. 2010).

\section{Laurencia okamurae Yamada}

Examined specimens: St. 1; NIBRAL0000117607 (22 Jul. 
2010), NIBRAL0000117608 (22 Jul. 2010), NIBRAL00 00121554 (14 Sep. 2010). St. 2; NIBRAL0000117606 (22 Jul. 2010). St. 3; NIBRAL0000117605 (21 Jul. 2010). St. 4; NIBRAL0000117311 (19 May 2010), NIBRAL00 00117609 (21 Jul. 2010), NIBRAL0000117610 (21 Jul. 2010), NIBRAL0000121566 (15 Sep. 2010). St. 5; NIB RAL0000117258 (31 Mar. 2010), NIBRAL0000117259 (31 Mar. 2010), NIBRAL0000117310 (19 May 2010).

\section{Laurencia pinnata Yamada}

Examined specimens: St. 1; NIBRAL0000117572 (22 Jul. 2010), NIBRAL0000117573 (22 Jul. 2010), NIBRA L0000121555 (14 Sep. 2010). St. 2; NIBRAL00001175 71 (22 Jul. 2010). St. 4; NIBRAL0000117570 (21 Jul. 2010). St. 5; NIBRAL0000117292 (19 May 2010).

\section{Laurencia venusta Yamada}

Examined specimens: St. 1; NIBRAL0000121556 (14 Sep. 2010), St. 2; NIBRAL0000121559 (14 Sep. 2010). St. 3; NIBRAL0000117537 (21 Jul. 2010), NIBRAL000 0121558 (14 Sep. 2010). St. 4; NIBRAL0000117536(21 Jul. 2010). St. 6; NIBRAL0000121557 (15 Sep. 2010).

\section{Neorhodomela munita (L.P.Perestenko) Masuda}

Examined specimens: St. 3; NIBRAL0000117530 (21 Jul. 2010). St. 5; NIBRAL0000117434 (19 May 2010), NIB RAL0000117436 (19 May 2010), NIBRAL0000117437 (19 May 2010). St. 6; NIBRAL0000117550 (21 Jul. 2010), NIBRAL0000117551 (21 Jul. 2010).

\section{Neosiphonia elongella (Harvey) M.S.Kim and I.K.Lee}

Examined specimens: St. 1; NIBRAL0000117122(30 Mar. 2010). St. 2; NIBRAL0000117443 (18 May 2010). St. 4; NIBRAL0000117440 (19 May 2010), NIBRAL00 00117442 (19 May 2010). St. 5; NIBRAL0000117123 (31 Mar. 2010), NIBRAL0000117438 (19 May 2010), NIB RAL0000117439(19 May 2010).

\section{Odonthalia corymbifera (S.G.Gmelin) Greville}

Examined specimens: St. 1; NIBRAL0000117207 (30 Mar. 2010), NIBRAL0000117208 (30 Mar. 2010), NIB RAL0000117209 (30 Mar. 2010), NIBRAL0000117418 (18 May 2010), NIBRAL0000117419 (18 May 2010), NIBRAL0000117711 (22 Jul. 2010), NIBRAL0000121 575 (14 Sep. 2010), NIBRAL0000121576 (14 Sep. 2010). St. 2; NIBRAL0000117205 (30 Mar. 2010), NIBRAL00 00117414 (18 May 2010), NIBRAL0000117415 (18 May 2010), NIBRAL0000117416 (18 May 2010), NIBRAL0
000117417 (18 May 2010), NIBRAL0000117709 (22 Jul. 2010), NIBRAL0000117710 (22 Jul. 2010), NIBRAL00 00117762 (22 Jul. 2010), NIBRAL0000121577 (14 Sep. 2010). St. 3; NIBRAL0000117204 (30 Mar. 2010), NIB RAL0000117206 (30 Mar. 2010), NIBRAL0000117411 (18 May 2010), NIBRAL0000117412 (18 May 2010), NI BRAL0000117413 (18 May 2010), NIBRAL000011770 7 (21 Jul. 2010), NIBRAL0000117708 (21 Jul. 2010), NI BRAL0000121578 (14 Sep. 2010), NIBRAL000012157 9 (14 Sep. 2010). St. 5; NIBRAL0000117210 (31 Mar. 2010), NIBRAL0000117407 (19 May 2010).

\section{Polysiphonia morrowii Harvey}

Examined specimens: St. 1; NIBRAL0000117175 (30 Mar. 2010). St. 2; NIBRAL0000117179 (30 Mar. 2010). St. 5; NIBRAL0000117177 (31 Mar. 2010), NIBRAL00 00117178 (31 Mar. 2010). St. 7; NIBRAL0000117176 (31 Mar. 2010).

\section{Symphyocladia latiuscula (Harvey) Yamada}

Examined specimens: St. 1; NIBRAL0000117121 (30 Mar 2010), NIBRAL0000117717 (22 Jul. 2010), NIBR AL0000117718 (22 Jul. 2010), NIBRAL0000117719 (22 Jul. 2010), NIBRAL0000121610 (14 Sep. 2010), NIBR AL0000121611 (14 Sep. 2010), NIBRAL0000121612 (14 Sep. 2010), NIBRAL0000121613 (14 Sep. 2010). St. 2; NIBRAL0000117715 (22 Jul. 2010), NIBRAL0000117 716 (22 Jul. 2010), NIBRAL0000121614 (14 Sep. 2010), NIBRAL0000121615 (14 Sep. 2010). St. 3; NIBRAL00 00117665 (21 Jul. 2010), NIBRAL0000117712 (21 Jul. 2010), NIBRAL0000117713 (21 Jul. 2010), NIBRAL00 00117714 (21 Jul. 2010), NIBRAL0000121616 (14 Sep. 2010), NIBRAL0000121617 (14 Sep. 2010). St. 4; NIB RAL0000117721 (21 Jul. 2010). St. 5; NIBRAL0000117 330 (19 May 2010, NIBRAL0000117331 (19 May 2010). St. 6; NIBRAL0000117720 (21 Jul. 2010), NIBRAL000 0121618 (15 Sep. 2010), NIBRAL0000121619 (15 Sep. 2010).

Order Corallinales

Family Corallinaceae

\section{Amphiroa ephedraea (Lamarck) Decaisne}

Examined specimens: St. 3; NIBRAL0000117203 (30 Mar. 2010).

\section{Bossiella cretacea (Postels and Ruprecht) H.W.Johansen}

Examined specimens: St. 1; NIBRAL0000117461 (18 May 2010), NIBRAL0000117462 (18 May 2010), NIB 
RAL0000121654 (14 Sep. 2010). St. 2; NIBRAL000011 7269 (30 Mar. 2010), NIBRAL0000117466 (18 May 2010), NIBRAL0000117469 (18 May 2010), NIBRAL0 000117756 (22 Jul. 2010), NIBRAL0000121652 (14 Sep. 2010). St. 3; NIBRAL0000117264 (30 Mar. 2010), NIB RAL0000117265 (30 Mar. 2010), NIBRAL0000117457 (18 May 2010), NIBRAL0000117459 (18 May 2010), NIBRAL0000117460 (18 May 2010), NIBRAL0000117 747 (21 Jul. 2010), NIBRAL0000121648 (14 Sep. 2010), NIBRAL0000121653 (14 Sep. 2010). St. 4; NIBRAL00 00117470 (19 May 2010), NIBRAL0000117471 (19 May 2010), NIBRAL0000117751 (21 Jul. 2010), NIBRAL00 00117752 (21 Jul. 2010), NIBRAL0000121649 (15 Sep. 2010), NIBRAL0000121650 (15 Sep. 2010), NIBRAL0 000121651 (15 Sep. 2010). St. 5; NIBRAL0000117260 (31 Mar. 2010), NIBRAL0000117261 (31 Mar. 2010). St. 6; NIBRAL0000117749 (21 Jul. 2010), NIBRAL000 0117750 (21 Jul. 2010), NIBRAL0000121655 (15 Sep. 2010).

\section{Corallina pilulifera Postels and Ruprecht}

Examined specimens: St. 1; NIBRAL0000117463 (18 May 2010), NIBRAL0000117464 (18 May 2010), NIB RAL0000117757 (22 Jul. 2010), NIBRAL0000117758 (22 Jul. 2010), NIBRAL0000117759 (22 Jul. 2010). St. 2; NIBRAL0000117467 (18 May 2010), NIBRAL0000 117468 (18 May 2010), NIBRAL0000117754 (22 Jul. 2010), NIBRAL0000117755 (22 Jul. 2010), NIBRAL00 00121659 (14 Sep. 2010), NIBRAL0000121660 (14 Sep. 2010). St. 3; NIBRAL0000117263 (30 Mar. 2010), NIB RAL0000117458 (18 May 2010), NIBRAL0000117746 (21 Jul. 2010), NIBRAL0000117748 (21 Jul. 2010), NIB RAL0000121656(14 Sep. 2010). St. 4; NIBRAL000011 7753 (21 Jul. 2010), NIBRAL0000121657 (15 Sep. 2010). St. 6; NIBRAL0000121658 (15 Sep. 2010). St. 7; NIBR AL0000117267 (31 Mar. 2010).

Family Hapalidiaceae

\section{Lithothamnion lemoineae Adey}

Examined specimens: St. 7; NIBRAL0000117268 (31 Mar. 2010).

\section{Synarthrophyton chejuensis J.H.Kim, H.Chung, D.S.Choi and I.K.Lee}

Examined specimens: St. 1; NIBRAL0000117465 (18 May 2010). St. 3; NIBRAL0000117262 (30 Mar. 2010), NIBRAL0000117760 (21 Jul. 2010), NIBRAL0000121 664 (14 Sep. 2010).

Order Halymeniales
Family Halymeniaceae

\section{Grateloupia filicina (J.V.Lamouroux) C.Agardh}

Examined specimens: St. 2; NIBRAL0000121606 (14 Sep. 2010). St. 4; NIBRAL0000117549 (21 Jul. 2010), NIBRAL0000121607 (15 Sep. 2010), NIBRAL0000121 609 (15 Sep. 2010). St. 6; NIBRAL0000121608 (15 Sep. 2010).

\section{Grateloupia lanceolata(Okamura) Kawaguchi}

Examined specimens: St. 4; NIBRAL0000117656 (21 Jul. 2010). St. 5; NIBRAL0000117200 (31 Mar. 2010).

\section{Grateloupia sparsa (Okamura) Chiang}

Examined specimens: St. 5; NIBRAL0000117247 (31 Mar. 2010).

\section{Polyopes lancifolius (Harvey) Kawaguchi and Wang}

Examined specimens: St. 4; NIBRAL0000117535 (21 Jul. 2010). St. 6; NIBRAL0000121647 (15 Sep. 2010).

$$
\begin{aligned}
& \text { Order Gigartinales } \\
& \text { Family Gigartinaceae }
\end{aligned}
$$

\section{Chondracanthus intermedius (Suringar) Hommersand in Hommersand, Guiry, Fredericq and Leister}

Examined specimens: St. 2; NIBRAL0000117319 (18 May 2010).

\section{Chondracanthus tenellus (Harvey) Hommersand in Hommersand, Guiry, Fredericq and Leister}

Examined specimens: St. 1; NIBRAL0000117544 (22 Jul. 2010), NIBRAL0000117545 (22 Jul. 2010), NIBRAL00 00117546 (22 Jul. 2010). St. 2; NIBRAL0000117580 (22 Jul. 2010). St. 3; NIBRAL0000117542 (21 Jul. 2010), NI BRAL0000117543 (21 Jul. 2010). St. 4; NIBRAL00001 17547 (21 Jul. 2010), NIBRAL0000121567 (15 Sep. 2010), NIBRAL0000121568 (15 Sep. 2010). St. 5; NIB RAL0000117174 (31 Mar. 2010). St. 6; NIBRAL000011 7581 (21 Jul. 2010), NIBRAL0000117582 (21 Jul. 2010), NIBRAL0000117583 (21 Jul. 2010).

\section{Chondrus ocellatus Holmes}

Examined specimens: St. 1; NIBRAL0000121545 (14 Sep. 2010). St. 2; NIBRAL0000121546 (14 Sep. 2010), 
NIBRAL0000121547 (14 Sep. 2010). St. 4; NIBRAL00 00117658 (21 Jul. 2010), NIBRAL0000117659 (21 Jul. 2010).

\section{Chondrus pinnulatus (Harvey) Okamura}

Examined specimens: St. 5; NIBRAL0000117307 (19 May 2010), NIBRAL0000117308 (19 May 2010), NIBR AL0000117309 (19 May 2010).

Family Kallymeniaceae

\section{Kallymenia crassiuscula Okamura}

Examined specimens: St. 2; NIBRAL0000117303 (18 May 2010), NIBRAL0000117304 (18 May 2010), NIBR AL0000117653 (22 Jul. 2010), NIBRAL0000117654 (22 Jul. 2010), NIBRAL0000117761 (22 Jul. 2010). St. 5; NI BRAL0000117302 (19 May 2010). St. 6; NIBRAL0000 117655 (21 Jul. 2010).

Family Phacelocarpaceae

\section{Phacelocarpus japonicus Okamura}

Examined specimens: St. 2; NIBRAL0000121541 (14 Sep. 2010). St. 4; NIBRAL0000121594 (15 Sep. 2010).

Family Phyllophoraceae

\section{Ahnfeltiopsis flabelliformis (Harvey) Masuda}

Examined specimens: St. 3; NIBRAL0000121544 (14 Sep. 2010). St. 5; NIBRAL0000117256 (31 Mar. 2010), NIBRAL0000117257 (31 Mar. 2010). St. 6; NIBRAL00 00117565 (21 Jul. 2010), NIBRAL0000117566 (21 Jul. 2010), NIBRAL0000121542 (15 Sep. 2010), NIBRAL0 000121543 (15 Sep. 2010).

\section{Ahnfeltiopsis paradoxus (Suringar) Masuda}

Examined specimens: St. 5; NIBRAL0000117305 (19 May 2010), NIBRAL0000117306 (19 May 2010).

Family Tichocarpaceae

\section{Tichocarpus crinitus (S.G.Gmelin) Ruprecht}

Examined specimens: St. 6; NIBRAL0000117548 (21 Jul. 2010).

Order Gelidiales

Family Gelidiaceae

\section{Gelidium amansii (J.V.Lamouroux)} J.V.Lamouroux

Examined specimens: St. 5; NIBRAL0000117255 (31 Mar. 2010).

\section{Gelidium vagum Okamura}

Examined specimens: St. 2; NIBRAL0000121569 (14 Sep. 2010).

Order Gracilariales

Family Gracilariaceae

\section{Gracilaria textorii (Suringar) De Toni}

Examined specimens: St. 4; NIBRAL0000117534 (21 Jul. 2010). St. 6; NIBRAL0000117533 (21 Jul. 2010).

\section{Gracilaria verrucosa (Hudson) Papenfuss}

Examined specimens: St. 2; NIBRAL0000117585 (22 Jul. 2010). St. 6; NIBRAL0000117584 (21 Jul. 2010).

\section{Order Nemaliales}

Family Liagoraceae

\section{Nemalion vermiculare Suringar}

Examined speciemens, St. 1; NIBRAL0000117541 (22 Jul. 2010).

Order Rhodymeniales

Family Champiaceae

\section{Champia parvula (C.Agardh) Harvey}

Examined specimens: St. 3; NIBRAL0000117669 (21 Jul. 2010).

Family Lomentariaceae

89. Gelidiopsis variabilis (Greville ex J.Agardh) Schmitz

Examined specimens: St. 1; NIBRAL0000117576 (22 Jul. 2010), NIBRAL0000117577 (22 Jul. 2010). St. 3; NIBR AL0000117574 (21 Jul. 2010). St. 4; NIBRAL00001175 75 (21 Jul. 2010).

\section{Lomentaria catenata Harvey}

Examined specimens: St. 3; NIBRAL0000117578 (21 Jul. 2010). 
Table 2. Species occurrence according to collection from Oho-ri.

\begin{tabular}{lcccrr}
\hline & Mar. & May & Jul. & Sep. & Total \\
\hline Chlorophyta & 6 & 8 & 6 & 4 & $13(13.8 \%)$ \\
Phaeophyta & 9 & 13 & 16 & 12 & $24(25.5 \%)$ \\
Rhodophyta & 29 & 29 & 38 & 24 & $57(60.6 \%)$ \\
\hline \multicolumn{1}{c}{ Total } & 44 & 50 & 60 & 40 & $94(100 \%)$ \\
\hline
\end{tabular}

Table 3. Inventory of marine algae of Oho-ri (species names were listed by alphabetical order under each phylum).

\begin{tabular}{|c|c|c|c|c|c|c|c|c|c|c|c|c|}
\hline \multirow{2}{*}{ Species } & \multicolumn{4}{|c|}{ Heukdo } & \multicolumn{4}{|c|}{ Goraebawi } & \multicolumn{4}{|c|}{ Juckdo } \\
\hline & Mar. & May & Jul. & Sep. & Mar. & May & Jul. & Sep. & Mar. & May & Jul. & Sep. \\
\hline \multicolumn{13}{|l|}{ Chlorophyta } \\
\hline Bryopsis hypnoides & & & & & & & & & & + & & \\
\hline Bryopsis plumosa & & & & & & & & + & & & & \\
\hline Chaetomorpha moniligera & & & & & & & & & & + & & \\
\hline Cladophora japonica & & & + & & & & + & & & & & \\
\hline Cladophora sakaii & & & + & & & & + & & & + & + & \\
\hline Cladophora stimpsonii & & & & & & & & & & + & & \\
\hline Codium arabicum & + & & + & + & & + & & + & & & & \\
\hline Codium fragile & & + & + & + & & & & & & + & + & + \\
\hline Monostroma grevillei & + & & & & + & & & & + & & & \\
\hline Ulva compressa & + & & & & & & & & & & & \\
\hline Ulva linza & + & & & & & & & & & & & \\
\hline Ulva pertusa & + & + & + & + & & + & + & & + & + & + & + \\
\hline Ulvaria obscura var. blyttii & + & + & + & & + & & & & + & + & + & \\
\hline \multicolumn{13}{|l|}{ Phaeophyta } \\
\hline Agarum clathratum & + & + & + & + & + & + & + & + & & & + & + \\
\hline Colpomenia bullosa & + & & & & & & & & & & & \\
\hline Colpomenia sinuosa & + & + & + & & & + & + & & + & + & + & \\
\hline Costaria costata & + & + & + & & + & + & + & & + & + & + & \\
\hline Desmarestia viridis & + & + & + & + & + & + & + & + & + & + & + & + \\
\hline Dictyopteris divaricata & + & + & + & + & & + & + & + & + & + & + & \\
\hline Dictyota dichotoma & & & + & + & & + & + & & & & + & \\
\hline Hydroclathrus clathratus & & + & & & & & & & & & & \\
\hline Leathesia difformis & & + & + & & & & & & & & + & \\
\hline Myagropsis myagroides & & + & & & & & & & + & + & & \\
\hline Papenfussiella kuromo & & & & & & & & & & + & & \\
\hline Saccharina japonica & & + & + & + & & & + & + & & + & + & \\
\hline Sargassum confusum & & & + & + & & & + & + & & & + & \\
\hline Sargassum hemiphyllum & & & & + & & & & + & & & + & + \\
\hline Sargassum horneri & & & + & + & & & & + & & & + & + \\
\hline Sargassum micracanthum & & & & & & & & & & & & + \\
\hline Sargassum miyabei & & & & + & & & & & & & + & \\
\hline Sargassum yezoense & & & & & & & & & & & + & \\
\hline Scytosiphon lomentaria & & + & & & & & & & & & & \\
\hline Spatoglossum crassum & & & & & & & & & & & + & \\
\hline Spatoglossum pacificum & & & + & & & & & & & & & \\
\hline Sporochnus radiciformis & + & & & & & & & & & & & \\
\hline Undaria pinnatifida & + & + & + & + & & & + & & + & + & + & \\
\hline Zonaria diesingiana & & & & + & & & & & & & & \\
\hline \multicolumn{13}{|l|}{ Rhodophyta } \\
\hline Acrosorium yendoi & & & & + & & & + & + & & & & + \\
\hline Aglaothamnion oosumiense & & & & & & & & + & & + & + & \\
\hline Ahnfeltiopsis flabelliformis & & & & & & & & + & + & & + & + \\
\hline Ahnfeltiopsis paradoxus & & & & & & & & & & + & & \\
\hline
\end{tabular}


Table 3. Continued.

\begin{tabular}{|c|c|c|c|c|c|c|c|c|c|c|c|c|}
\hline \multirow{2}{*}{ Species } & \multicolumn{4}{|c|}{ Heukdo } & \multicolumn{4}{|c|}{ Goraebawi } & \multicolumn{4}{|c|}{ Juckdo } \\
\hline & Mar. & May & Jul. & Sep. & Mar. & May & Jul. & Sep. & Mar. & May & Jul. & Sep. \\
\hline \multicolumn{13}{|l|}{ Rhodophyta (continued) } \\
\hline Amphiroa ephedraea & & & & & + & & & & & & & \\
\hline Bonnemaisonia hamifera & + & + & + & + & + & + & + & + & + & + & + & \\
\hline Bossiella cretacea & + & + & + & + & + & + & + & + & + & + & + & + \\
\hline Ceramium boydenii & & & & & & & & & & & + & \\
\hline Champia parvula & & & & & & & + & & & & & \\
\hline Chondracanthus intermedius & & + & & & & & & & & & & \\
\hline Chondracanthus tenellus & & & + & & & & + & & + & & + & + \\
\hline Chondria crassicaulis & + & & + & & & & & + & & + & + & + \\
\hline Chondrus ocellatus & & & & + & & & & & & & + & \\
\hline Chondrus pinnulatus & & & & & & & & & & + & & \\
\hline Chrysymenia wrightii & & & + & + & & & + & + & + & + & + & \\
\hline Corallina pilulifera & & + & + & + & + & + & + & + & + & & + & + \\
\hline Dasya collabens & & & & & & & & & + & & & \\
\hline Dasya villosa & & & & & & & & & & + & & \\
\hline Delesseria serrulata & + & + & & & + & & & & + & + & & \\
\hline Delisea pulchra & & + & & & + & & & & + & + & & \\
\hline Erythroglossum minimum & & + & + & + & + & + & & & & & & \\
\hline Gelidiopsis variabilis & & & + & & & & + & & & & + & \\
\hline Gelidium amansii & & & & & & & & & + & & & \\
\hline Gelidium vagum & & & & + & & & & & & & & \\
\hline Gracilaria textorii & & & & & & & & & & & + & \\
\hline Gracilaria verrucosa & & & + & & & & & & & & + & \\
\hline Grateloupia filicina & & & & + & & & & & & & + & + \\
\hline Grateloupia lanceolata & & & & & & & & & + & & + & \\
\hline Grateloupia sparsa & & & & & & & & & + & & & \\
\hline Herpochondria elegans & & + & & & & & & & + & & & \\
\hline Heterosiphonia japonica & & & + & + & + & + & + & & & + & + & \\
\hline Heterosiphonia pulchra & + & + & + & + & & + & + & + & & + & + & + \\
\hline Kallymenia crassiuscula & & + & + & & & & & & & + & + & \\
\hline Laurencia intricata & & & & & & & & & & & + & \\
\hline Laurencia nipponica & & & & & & & & & & & + & \\
\hline Laurencia okamurae & & & + & + & & & + & & + & + & + & + \\
\hline Laurencia pinnata & & & + & + & & & & & & + & + & \\
\hline Laurencia venusta & & & & + & & & + & + & & & + & + \\
\hline Lithothamnion lemoineae & & & & & & & & & + & & & \\
\hline Lomentaria catenata & & & & & & & + & & & & & \\
\hline Lomentaria hakodatensis & & & & & & & & & & + & & \\
\hline Nemalion vermiculare & & & + & & & & & & & & & \\
\hline Neoholmesia japonica & + & + & + & + & + & + & + & + & & & & \\
\hline Neorhodomela munita & & & & & & & + & & & + & + & \\
\hline Neosiphonia elongella & + & + & & & & & & & + & + & & \\
\hline Odonthalia corymbifera & + & + & + & + & + & + & + & + & + & + & & \\
\hline Phacelocarpus japonicus & & & & + & & & & & & & & + \\
\hline Polyopes lancifolius & & & & & & & & & & & + & + \\
\hline Polysiphonia morrowii & + & & & & & & & & + & & & \\
\hline Pterothamnion yezoense & & + & & & & & & & + & + & & \\
\hline Pyropia tenera & & & & & & & & & + & + & & \\
\hline Rhodymenia intricata & & & + & & & & & & & & & \\
\hline Sorella repens & & & & & & & + & & & & & \\
\hline Sparlingia pertusa & + & + & & & + & + & + & & + & + & & \\
\hline Symphyocladia latiuscula & + & & + & + & & & + & + & & + & + & + \\
\hline Synarthrophyton chejuensis & & + & & & + & & + & + & & & & \\
\hline Tichocarpus crinitus & & & & & & & & & & & + & \\
\hline
\end{tabular}




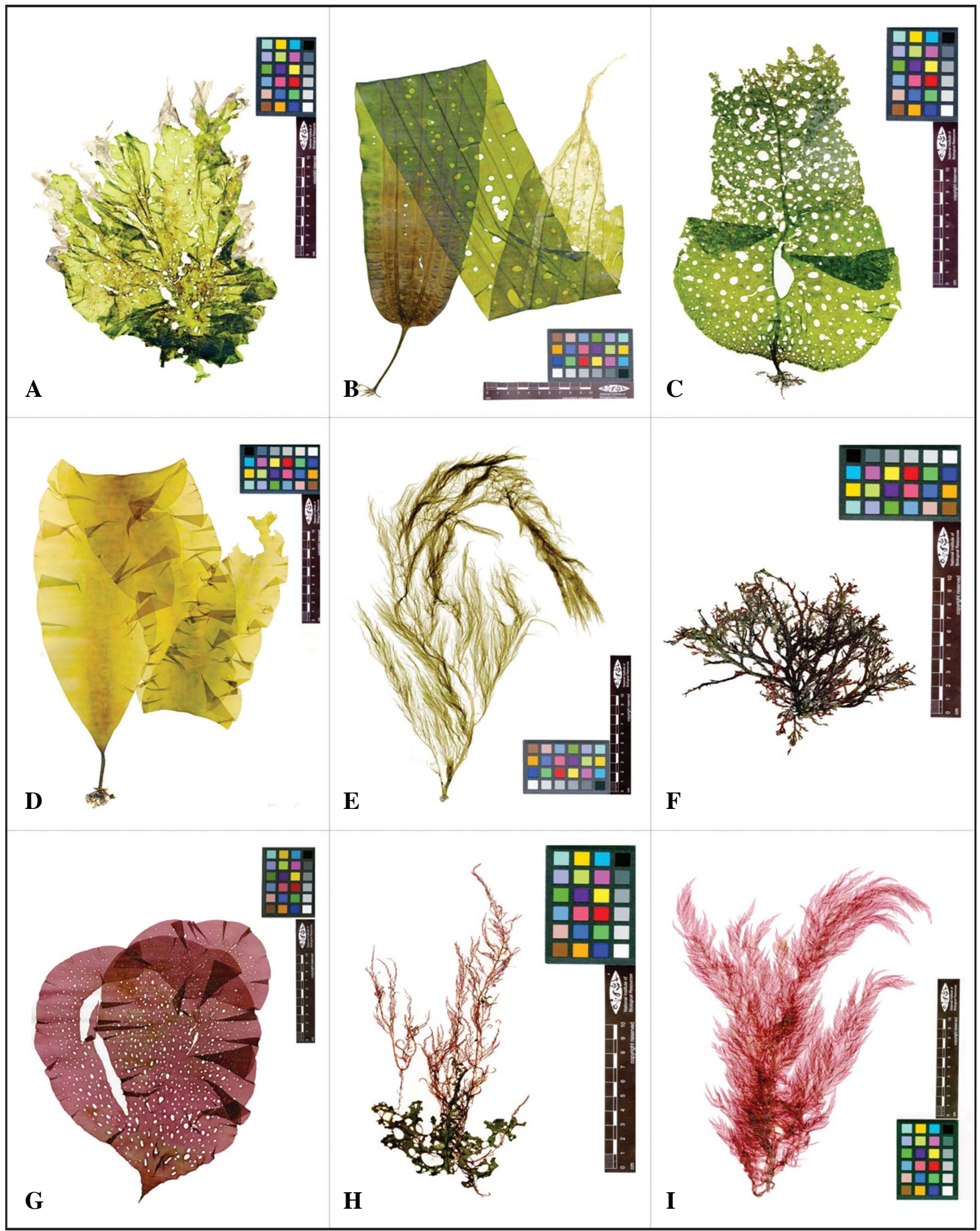

Fig. 2. Some selected marine algal specimens at Oho-ri in 2010. A. Ulvaria obscura var. blyttii from Juckdo in May. B. Costaria costata from Heukdo in May. C. Agarum clathratum from Heukdo in March. D. Saccharina japonica from Heukdo in May. E. Desmarestia viridis from Heukdo in March. F. Odonthalia corymbifera from Heukdo in March. G. Sparlingia pertusa from Heukdo in March. H. Bonnemaisonia hamifera from Heukdo in May. I. Delesseria serrulata from Juckdo in March. 


\section{Lomentaria hakodatensis Yendo}

Examined specimens: St. 4; NIBRAL0000117286 (19 May 2010).

Family Rhodymeniaceae

\section{Chrysymenia wrightii(Harvey) Yamada}

Examined specimens: St. 1; NIBRAL0000117647 (22 Jul. 2010), NIBRAL0000117648 (22 Jul. 2010). St. 2; NIBR AL0000117646 (22 Jul. 2010), NIBRAL0000121630 (14 Sep. 2010), NIBRAL0000121631 (14 Sep. 2010). St. 3; NIBRAL0000117644 (21 Jul. 2010), NIBRAL0000117 645 (21 Jul. 2010), NIBRAL0000121632 (14 Sep. 2010). St. 4; NIBRAL0000117430 (19 May 2010), NIBRAL00 00117431 (19 May 2010), NIBRAL0000117569 (21 Jul. 2010). St. 5; NIBRAL0000117426 (19 May 2010), NIB RAL0000117427 (19 May 2010), NIBRAL0000117428 (19 May 2010), NIBRAL0000117429 (19 May 2010). St. 7; NIBRAL0000117253 (31 Mar. 2010).

\section{Rhodymenia intricata (Okamura) Okamura}

Examined specimens: St. 1; NIBRAL0000117660 (22 Jul. 2010).

\section{Sparlingia pertusa (Postels and Ruprecht) G.W.Saunders, I.M.Strachan and Kraft}

Examined specimens: St. 1; NIBRAL0000117397 (18 May 2010), NIBRAL0000117398 (18 May 2010). St. 2; NIBRAL0000117232 (30 Mar. 2010), NIBRAL0000117 392 (18 May 2010), NIBRAL0000117394 (18 May 2010), NIBRAL0000117395 (18 May 2010). St. 3; NIBRAL00 00117231 (30 Mar. 2010), NIBRAL0000117391 (18 May 2010), NIBRAL0000117657 (21 Jul. 2010). St. 5; NIBR AL0000117229 (31 Mar. 2010), NIBRAL0000117230 (31 Mar. 2010), NIBRAL0000117387 (19 May 2010), NIBRAL0000117388 (19 May 2010).

The total species composition was as follows: green algae, 13 species (13.1\%); brown algae, 24 species (24.2 $\%$ ); and red algae, 62 species (62.6\%). The species diversity was the highest in June (63 species), followed by that in May (52), March (45), and September (43) (Table 2). The occurrence of species according to the survey sites and month was shown in Table 3. During the survey, 12 housekeeping species were identified; Ulva pertusa, Desmarestia viridis, Bonnemaisonia hamifera, Bossiella cretacea, Chrysymenia wrightii, Corallina pilulifera, Heterosiphonia japonica, Heterosiphonia pulchra, Laurencia okamurae, Neoholmesia japonica, Odonthalia corymbifera, and Symphyocladia latiuscula. Some selected specimens of marine algae were presented in Fig. 2.

Marine algal flora of the northern coast of the East Sea have been reported at the several sites, 39 species at Gonghyunjin (Lee, 1991), 100 species in Juckdo, Bongpori (Nam, 1986), 114 species at Sokcho (Boo and Lee, 1986; Lee, 1991; Lee et al., 1993), and 110 species at Anin in Kangneung (Kim et al., 1983; Boo, 1985; Lee, 1991). Koh (1983) stated that Sargassum species among brown algae, including $S$. confusum and $S$. horneri had occurred in Juckdo throughout survey period of the year 1982 to 1983. However, this survey showed that their occurrences were observed only in July and September of 2010.

In this study, 94 species of marine algae were collected from Oho-ri. The species list and its voucher specimens would be clue of the marine algae diversity changes to the spatio-temporal climate change.

\section{ACKNOWLEDGMENTS}

We thank referees for providing advice and Hyun Soo Kim and Cho-A Kim for helping sample collection. This research was supported by grants from "The Survey on Species Diversity of Strategic Regions Project" by the National Institute of Biological Resources, the Ministry of Environment, Korea.

\section{REFERENCES}

Boo, S.M. 1985. Vertical distribution patterns of the intertidal algal community on a Kangreung rocky shore, east coast of Korea. Proc. Nat. Sci. Res. Inst. KANU 1:46-53.

Boo, S.M. and I.K. Lee. 1986. Studies on benthic algal community in the East Coast of Korea 1. Floristic composition and periodicity of a Sokcho rocky shore. Korean J. Phycol. 1:107-116.

Kang, J.W. 1968. Illustrated encyclopedia of fauna and flora of Korea vol. 8. Marine algae. Ministry of Education. pp. 465.

Kim, H.S., I.K. Lee, C.H. Koh, I.H. Kim, Y.B. Suh and N.K. Sung. 1983. Studies on the marine benthic communities in inter- and subtidal zones I. Analysis of benthic community structures at Aninjin, Eastern Coast of Korea. Proc. Coll. Natur. Sci., SNU 8:71-108.

Koh, C.H. 1983. Community Structure and Productivity of Phyto benthos in Juckdo (Eastern Coast of Korea). II. Seasonal changes of algal vegetation in relation to annual growth of large brown algae. Korean Journal of Botany 26:181-190.

Koh, C.H. and N. Sung. 1983. Community structure and productivity of phytobenthos in Juckdo (Eastern Coast of Korea). I. Benthic marine algal vegetation and its environment. Korean Journal of Botany 26:119-130. 
Lee, J.W. 1991. Community structure and geographical distribution of intertidal benthic algae in the East Coast of Korea. Ph.D. thesis. Seoul National Univ., Seoul. pp. 210.

Lee, J.W., H.B. Lee and I.K. Lee. 1993. A study on the community structure of intertidal marine benthic algae in the East coast of Korea I. An intertidal marine benthic algal community at Sokcho. Korean J. Phycol. 8:67-75.

Lee, Y. 2008. Marine Algae of Jeju. Academic Science. pp. 477.

Nam, K.-W. 1986. On the marine benthic algal community of Chuckdo in Eastern Coast of Korea. Korean J. Phycol. 1:185-202.

National Institute of Biological Resources. 2009. Optimization and standardization of storage and management process of biological resource collections at the National Institute of Biological Resources (II). pp. 225.

Yoshida, T. 1998. Marine algae of Japan. Uchida Rokakuho Publishing Co. Tokyo.

Submitted: December 23, 2013, Accepted: February 17, 2014 